
\title{
25 Research Soure \\ What Happened to the Laboratory Animals during COVID-19 Pandemic lockdown? India Answers.
}

Harikrishnan, V.S.

SCMST

\section{Sarita Jena}

Institute of Life Sciences

Shika Yadav

National Institute of Biologicals

Kalidas Kohale

Tata Institute of Fundamental Research

Jerald Mahesh Kumar

CCMB: Centre for Cellular and Molecular Biology CSIR

Perumal Nagarajan ( $\nabla$ nagarajan@nii.ac.in )

National Institute of Immunology https://orcid.org/0000-0003-4557-4633

\section{Research Article}

Keywords: Survey, India, COVID19, Laboratory animals, Research

Posted Date: August 2nd, 2021

DOl: https://doi.org/10.21203/rs.3.rs-762392/v1

License: (c) (i) This work is licensed under a Creative Commons Attribution 4.0 International License.

Read Full License 


\section{Abstract}

Background : COVID-19 imposed euthanasia of research animals and partial or complete closure of laboratory animal facilities across the world in the recent. In an attempt to find out the impact on laboratory animals, a survey was conducted ensuring the voluntary participation of institutions from different regions of India.

Results : A total of 56 facilities participated from 14 states and union territories. From their responses $94.64 \%$ of the facilities operated either fully or partly with $12.5 \%$ operating completely. $62.5 \%$ had no interruption in receiving essential supplies and $16.1 \%$ operated with complete man-power. $19.64 \%$ paused breeding completely and $53.57 \%$ partially. Besides strict lockdown, $95.83 \%$ of the institutes were able to provide veterinary care. $58.93 \%$ of the institutions conducted online IAEC meetings indicative of continuing research activities. Many facilities reported morbidities (64.29\%) and mortality (8.93\%). To optimize resources $41 \%$ reported euthanasia of animals.

Conclusion : Statistical analysis revealed a significant association of lockdown associated reduced manpower and impaired essential-supplies with these observed eventualities. In conclusion, even though the lockdown had created a partial disruption of activities, operations and research went on in the majority of the facilities in India.

\section{Background}

COVID-19 pandemic has disrupted normal working patterns in all areas including laboratory animal facilities across the globe. Unlike most of the other working areas, animal facilities require year-round human intervention to facilitate the care, husbandry and survival of various experimental animal species. The working of animal facilities to uphold research activities is also of equal importance. The urgent mandate of COVID-19 vaccine development [1] and other relevant products required uninterrupted functioning of research facilities. Tactlessly, several thousands of mice had been euthanized as per press reports in research laboratories in the United states and across the European union whereas many research laboratories were forced to shut down their operations [2].This was owing to a lack of trained staff for attending the animals owing to the lock-downs, broken essentials supply and the alarmingly high hospitalizations and havoc witnessed by many nations during the outbreak. Animal health was also affected due to the immediate repercussions related to COVID-19 [3]. Like the US and EU, India also led their COVID-19 vaccine research and development in the run to find an elixir to harness the pandemic. The actual scenario of activities and functioning of the animal facilities in India during the lockdown has yet not unravelled. This data along with the lessons learned are of interest as well as important for planning of activities and welfare protocols to meet similar crises in the future.

A survey was planned, with confidentiality assured to respondents and questionnaires were sent to 56 organizations consisting of academic/research institutions, medical, veterinary, pharmacy colleges and CROs having animal facilities across India and confidentiality was assured to all the respondents. The 
survey included animal facilities from the East, West, North, South and Central parts of the Indian mainland. The Committee for the Purpose of Control and Supervision of Experiments on Animals (CPCSEA), the statutory authority that controls research using animals in India made several favourable that include digitalizing the Institutional Animal Ethics Committee (IAEC) meetings and including animal feed and bedding as an essential commodity and thereby prioritising its transport across India. Besides, the veterinary care and animal facility staff were considered as frontline healthcare workers and thereby excluding them from curfews and lockdowns.

Despite the above positive efforts by the government to overcome the situation, few hurdles still pertained owing to travel restrictions/difficulty in obtaining raw materials to manufacture feed by manufacturers and also finding transportation system to deliver feed, bedding material and animals specifically owing to restrictions on entry and exit within states in addition to the quarantine regulations that followed had an impact on feed, bedding material and animal supplies. It was hypothesised that the supply chain would have been affected leading to euthanasia of laboratory animals, reduction in the research activities and other catastrophes associated with the impaired operations like the incidence of morbidities and mortalities in colonies. It was also hypothesised that; the majority of the institutions would have partly euthanized animals to optimize available resources and to meet reduced man-power availability. Based on the above hypothesis the survey was conducted to know the actual situation of the animal facilities during this pandemic.

\section{Results}

From the survey on 56 institutions, a total of 4,38,582 animals were used for research during 2019-20 comprising $83.9 \%$ mice, $9.64 \%$ guinea pigs, $5.31 \%$ rats, $0.26 \%$ other rodents, $0.48 \%$ of rabbits, $0.35 \%$ of large animals, $0.04 \%$ of NHPs and $0.05 \%$ of birds. Four institutions reported that they didn't house any live animals during 2019-20. (Figure 1)

\section{Shut down status and supplies during Lockdown:}

During the lockdown period, $12.5 \%$ and $82.14 \%$ of the facilities were fully and partially operational respectively, whereas $5.36 \%$ of facilities were completely closed down (Figure 2).

\section{Cease of essential supplies:}

$18.6 \%$ of the institutions were fully functional with the availability of essential supplies. However, $63.4 \%$ of facilities had uninterrupted supplies of critical items such as feed, bedding and drugs. Thirty-seven and a half percent of the facilities had no supplies and they could manage with the available resources. To cope with the situation, 17 institutions started to purchase and use locally available animal feed and 8 institutions found a local alternative for bedding material while 35 institutions didn't find a need to use local alternatives for any of the essential supplies. Seven percent of the facilities that did not breed animals in-house for research, procured animals from the local areas. The availability of drugs for 
veterinary use was not a problem and only 3 institutions started to procure drugs available locally (Figure 3,4).

\section{Euthanasia of animals to manage Resources:}

As per the survey, more than $50 \%(58.92 \%)$ of the facilities tried to avoid euthanasia and were able to manage their daily operations with the available resources. However, $41.1 \%$ of the facilities reported partial euthanasia of the animals to optimize the available resources anticipating scarcity of in their vicinity and reduced manpower (Figure 5).

\section{Manpower available during lockdown to cater animal facility:}

As per the Department of Personnel and Training, Govt. of India's (11013/9/2014-Estt.A-III dated 23rd April 2020), institutions had the permission to operate with one-third staff strength. $58.9 \%$ of the institutions had reduced the staff strength to $50 \%$ to minimize risks by exposing all the staff at the same time to the possible COVID19 infection. Few (16.1\%) of the facilities operated with full staff strength whereas $25 \%$ of the institutions reduced staff strength where they retained over $50 \%$, but without laying off (Figure 6).

\section{Reduction in breeding program:}

It was noticed that $19.64 \%$ of the facilities stopped breeding completely and $53.57 \%$ partially whereas $16.07 \%$ of the facility did not change their breeding program and continued to breed as was being done before the lockdown. The remaining $10.07 \%$ of the institutions mentioned that the question on breeding reduction did not apply to them (Figure 7).

\section{Veterinary Care:}

The health of the animals was given priority and a majority of the institutes (95.83\%) provided veterinary care to animals. While $76.19 \%$ of the institutions provided full-time Veterinarians, in $19.64 \%$ the availability of veterinarians was on-call and only $8 \%$ of the facilities were unable to provide veterinary care (Figure 8).

\section{IAEC meeting for protocols involving animal research:}

Indian research institutions continued to perform research and hence, $58.93 \%$ of the institutions made use of the CPCSEA order to conduct online IAEC meetings and the rest of the institutes $(41.07 \%)$ did not perform IAEC meetings during the lockdown (Figure 9).

\section{Morbidity and Mortality reported during lockdown period:}

Animal morbidities and mortalities were reported in $64.29 \%$ and $8.93 \%$ of the facilities respectively. This could be associated with reduced manpower or compromised essential supplies in many facilities due to complete lockdown (Figure-10 and 11). Most of the institutions opined that regional/local care-taking 
staff from near-by areas shall be posted in facilities to take care of animals during similar crises in the future. A significant association of morbidity, mortality, euthanasia or reduced breeding owing to COVID19 lockdown could be observed from the Chi-squared test $(\chi 2(1, N=54)=4.103, P=0.0214)$.

Most of them opined that there should be surplus stock of critical supplies such as feed and bedding in the institutions. Also, cryopreservation facility, regional centralization by creating common animal facilities in all institutions in respective cities and availing local supplies were the other suggestions on how to tide over similar situations in the future.

\section{Discussion}

The species-wise data on animals used per year are annually published by at least 37 nations including Great Britain [4] Australia, Bulgaria, Japan, the United States of America [5]and the European Union [6] The annual laboratory animal usage statistics in Indian laboratories were also projected for the year 2015 using a statistical model. However, annual species wise usage data is extremely rare or lacking from India who is the largest provider of generic drugs globally (https://www.ibef.org/industry/pharmaceuticalindia.aspx) that ranked $11^{\text {th }}$ in global pharmaceutical exports. India provides over $50 \%$ of the vaccines of the global demand. Using the present survey sample from 56 institutions, it could be projected with a proportionate projection that, in 2018-2019, in India with 1748 CPCSEA registered animal facilities used a total of $1,14,81,488$ mice, $13,19,459$ Guinea pigs, 7,26,637 rats, 34,991 other rodents, 66,018 rabbits, 7,210 birds, 5,712 NHP's and 47,945 large animals for research and education. Further, a total of 13,689,460 animals are expected to be used in India for research, testing and education in the year 2020, As per the report from the commission to the European parliament and the council 2019-report on the statistics on the use of animals for scientific purposes in the Member States of the European Union in 2015-2017 a total of 9,590,379 animals in the year 2015, 9,817,946 in 2016 and 9,388,162 during 2017 were used for the research and education. in the European Union. The percentage of mice usage was $61 \%$ as reported by EU in 2017 against $83.9 \%$ as estimated by the present study in India. Similarly, the percentage of rats used by the EU was $12 \%$ (against the $5.31 \%$ as estimated from India now from this survey), birds are $6 \%$ against the $0.05 \%$ as estimated from the survey. This will be one of the initial attempts to estimate the species wise percentage and the total number of animals used in India for research, testing and education. However, this is an estimate based on data from 56 institutes only and the data based on actual usage will be more precise.

The road/rail /air connectivity between different regions in India varies considerably. Within the states themselves, due to geographical diversities, connectivity and accessibility differ; as many areas don't have rail or air access. Hence, the data on receipt of essential supplies during the lock-down as such is of limited value and will have high variability. It is impossible to put light into each particular area in the Indian mainland on this issue and so a general prediction is being attempted. However, one respondent commented that the facility had to pay additional costs for procuring supplies from the usual vendor/s. Most of the affected facilities were dependent on local alternatives. Among the supplies that were disrupted, the animal feed was procured by most of these facilities followed by bedding, animals and 
drugs from local sources. Further, many animal facilities anticipated an interruption in supply lines and resorted to euthanasia to facilitate smooth utilization of available resources. The same could be the reason behind the reduction of purpose-breeding of animals by some of the facilities. Limited availability of specialized and trained care-taking staff also may have contributed to euthanasia and reduced breeding of animals in facilities across India. The interrupted supply of routinely used feed and bedding together with reduced staff-strength would have led some of the animal facilities to report morbidities and mortalities in animals. The statistical test done reinforces the association between all these factors owing to lockdown like reduced staff strength and impaired essential supplies to mortality, morbidity, reduced breeding and euthanasia to optimize services. It is also worthwhile to note that veterinary care was available full-time or on-call in the majority of the facilities.

However, the modified amendment brought by the statutory body in India, CPCSEA to conduct online IAEC meetings was utilized by the majority of the facilities and this indicates that research and testing during COVID-19 pandemic was on the roll even when adversities of the pandemic struck animal facilities across India. The thoughtfulness of CPCSEA to implement online meetings towards aiding for the care of animals and continuation of the experimentation and declaring animal feed and bedding as essential items to ensure transport across states during lockdown is commendable. Despite the financial slow down imparted by COVID-19 pandemic on the Indian economy [7], the facility management strived well to keep the research going and at the same time take good care of the research animals' welfare. India was also employee-friendly, providing salaries and avoiding layoffs during this global crisis and this was empowered through a Union government order as well. The responses from the survey indicated that the use of local products and surplus storage of critical items like feed and bedding together with local staff who are residing near to the facilities can be the key in handling similar crises in the future.

\section{Pandemic Management Strategies for Animal Facilities}

To cope up with the adversities faced owing to the lockdown due to COVID-19, Indian laboratory animal facilities tried their best to safeguard the well-being of animals and personnel involved. Even with disaster management plans in place, the extraordinary crisis pushed the animal facilities to make situational decisions and on the spot planning. The strategies were emphasized to take care of eventualities concerning the safety, health and well-being of research animals and animal caretaking personnel. These anticipated measures aiming to negotiate with such eventualities in the future are enlisted as follows:

- Stocking of available essentials like feed, bedding, sanitization items, drugs as much as possible to manage with an unpredictable duration of lockdown.

- Optional utilization of locally available substitute of essentials as back up.

- Optimal utilization of available resources shall be made. Instead of disposable PPE, reusable autoclavable PPE can be used. Flexibility in procedures like cage changing frequency with spot changing option for few dirty cages and reduced frequency of facility cleaning and sanitization.

- The animal stock and breeding shall be temporarily reduced to optimize available resources. 
- Reduction in non-essential research activities, procedures and prioritization of routine husbandry practices for stocked animals to carry out daily activities with the reduced manpower.

- Virtual meeting and orientation shall be practised for researchers for the holding of and management of ongoing animal experiments

- Transportation of animals to and from the facilities shall be stopped except in COVID-related or another important ongoing research.

\section{Conclusion}

Research using animals was taken forward by Indian institutions during the pandemic crisis even though the animals were identified with a rise in morbidities and mortality. The supply chain for essential items, in general, was intact in India. Veterinary care was available during the lockdown. Reduced staff strength and impaired essential supplies were associated with animal mortality, morbidity, reduced breeding and euthanasia to optimize services during the lockdown.

\section{Methods}

The survey was prepared in English and questionnaires were sent to research animal facility managers via e-mail and assurance on confidentiality statement was also given; that respondent's anonymity will be maintained. These contacts were obtained from their personal and professional accomplices by the authors who were members of the Indian association working for the welfare of laboratory animals. The survey that started on October 10th, 2020 and was closed by January 26th, 2021 consisted of eleven questions and a space for suggestions to opine towards how to tackle similar situations. The survey includes the following parameters - a)species wise usage during the financial year 2019-2020, b) whether the facility was completely or partly shut-down, c)availability of essential supplies- feed, bedding, animals and drugs) local alternatives availed to overcome the shortage of essential supplies, e)euthanasia in connection to lockdown to optimize resources, f) availability of man-power, interruptions in availing Veterinary care, g)reduction in the breeding to reduce production, $h$ ) online IAEC meetings held j) if any mortalities or morbidities detected owing to lockdown. Since this survey was not using any animals for generating data, approval from the ethical committee approval was not sought by the authors. It was ensured that sending the survey to more than one person from the same institution was avoided and an agreement on maintenance of respondent's confidentiality was offered.

\section{Statistical Analysis}

Microsoft Excel was used to find out the species wise percentage of animals used and the percentages for questions asked for each parameter. Parts of whole graphs were made using GraphPad Prism version 9.0.0 for Windows, GraphPad Software, San Diego, California USA, www.graphpad.com. Chi-squared test of independence was performed to assess the association of morbidity, mortality, euthanasia or reduced breeding due to lockdown. $P>0.05$ was considered as statistically significant. 


\section{Declarations}

\section{Data availability statement:}

The authors confirm that the data supporting the findings of this study are available and given on request

Funding: No funding received for this study

Conflict of Interest: All authors declare NO conflict of Interest

Authors' contributions: All Authors contributed equally

Animal Ethics Approval : Since this survey was not using any animals for generating data, approval from the ethical committee approval was not sought by the authors.

Acknowledgement: The authors wish to thank to the participating Institute and the members who provided this data.

\section{References}

1. Kumar S, Yadav PK, Srinivasan R, Perumal N. Selection of animal models for COVID-19 research. VirusDisease. 2020 Dec;31(4):453-8.

2. De Briyne N, Dalla Villa P, Ellis D, Golab G, Gruszynski K, Hammond-Seaman A, Moody S, Noga Z, Pawloski E, Ramos M, Simmons H. Overcoming the impact of Covid-19 on animal welfare: COVID-19 Thematic Platform on Animal Welfare.

3. Gortázar C, de la Fuente J. COVID-19 is likely to impact animal health. Prev Vet Med. 2020 Jul;1:180:105030.

4. Hudson-Shore M. Statistics of Scientific Procedures on Living Animals Great Britain 2015Highlighting an ongoing upward trend in animal use and missed opportunities for reduction. Alternatives to Laboratory Animals. 2016 Dec;44(6):569-80.

5. Taylor K, Alvarez LR. An estimate of the number of animals used for scientific purposes worldwide in 2015. Altern Lab Anim. 2019 Nov;47(5-6):196-213.

6. Busquet F, Kleensang A, Rovida C, Herrmann K, Leist M, Hartung T. New European Union statistics on laboratory animal use-what really counts!. ALTEX-Alternatives to animal experimentation. 2020 Mar 31;37(2):167-86.

7. Dev SM, Sengupta R. Covid-19: Impact on the Indian economy. Mumbai April: Indira Gandhi Institute of Development Research; 2020 Apr.

\section{Figures}




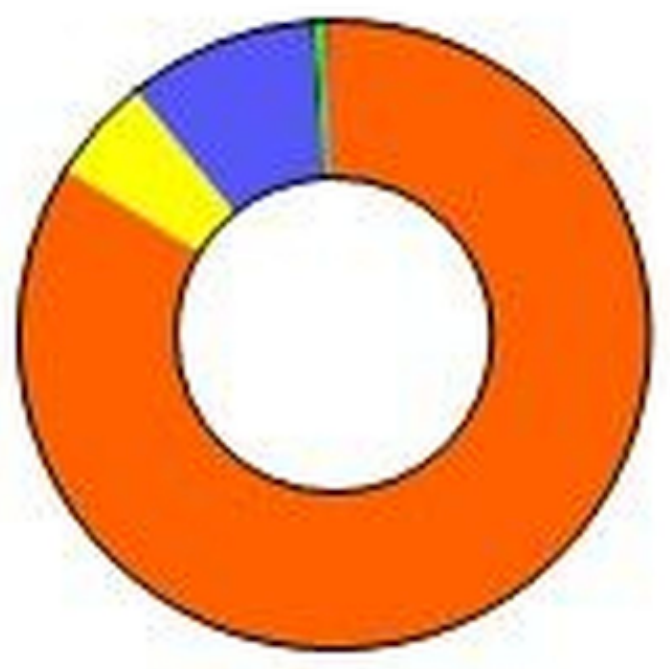

$\square 83.87 \% 387828$ Mice

$\square 5.31 \% 23279$ Rats

9.64\% 42271 Guinea Pigs

- $0.28 \% 1121$ Cther Rodents

$\square 0.48 \% 2115$ Rabbits

$\square 0.04 \% 183$ Non Human Primates

$0.35 \% 1536$ Large animals

$0.05 \% 231$ Birds

Fig-1: Specieswise percentage of animals used in India 2019-2020

Figure 1

Species wise percentage of Laboratory animals used in 2019 -2020 from 56 participated Institute

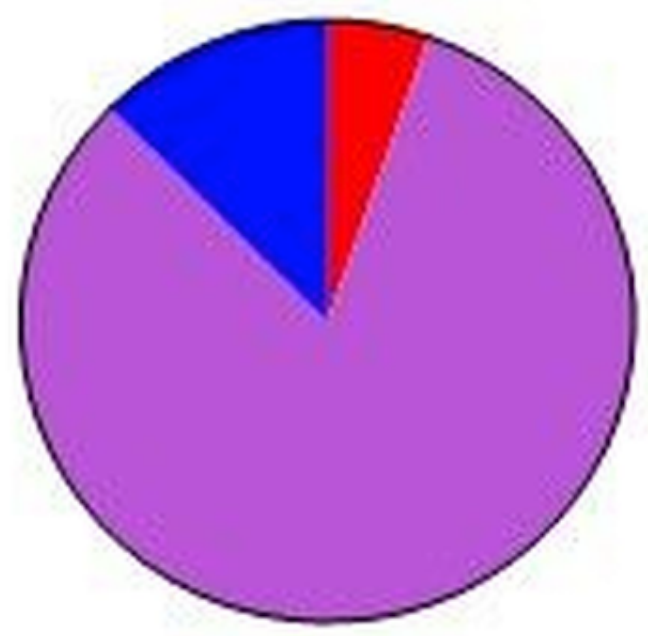

曰 $5.36 \% 3$ Total shutdown

曰 $82.14 \%$ 46 Partial Shutdown

- $12.50 \% 7$ Fully operational

Fig-2: Shut-down status and supplies during lockdown

Figure 2 


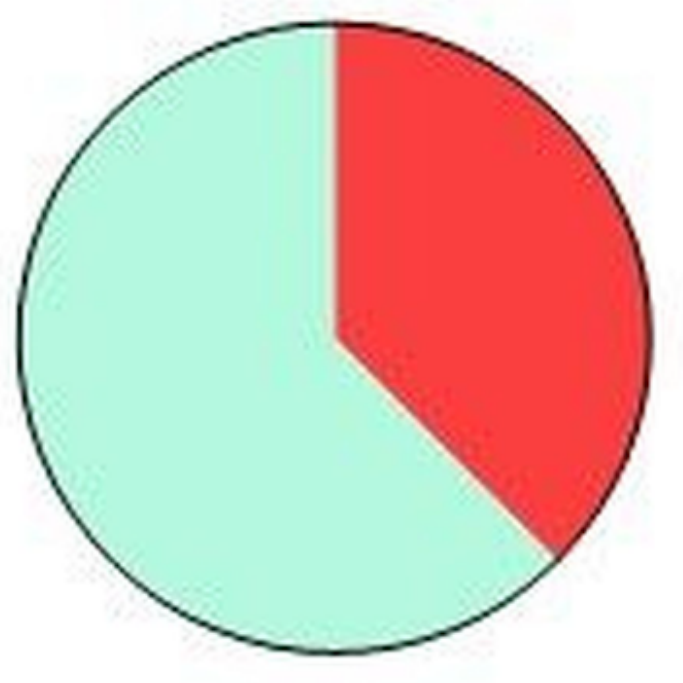

\section{曰 $37.50 \% 21$ Cease of supplies \\ $\square 62.50 \% 35$ Uninterrupted supplies}

Fig-3: Cease of Essential Supplies

\section{Figure 3}

Pie diagram representing cease of essential supplies 


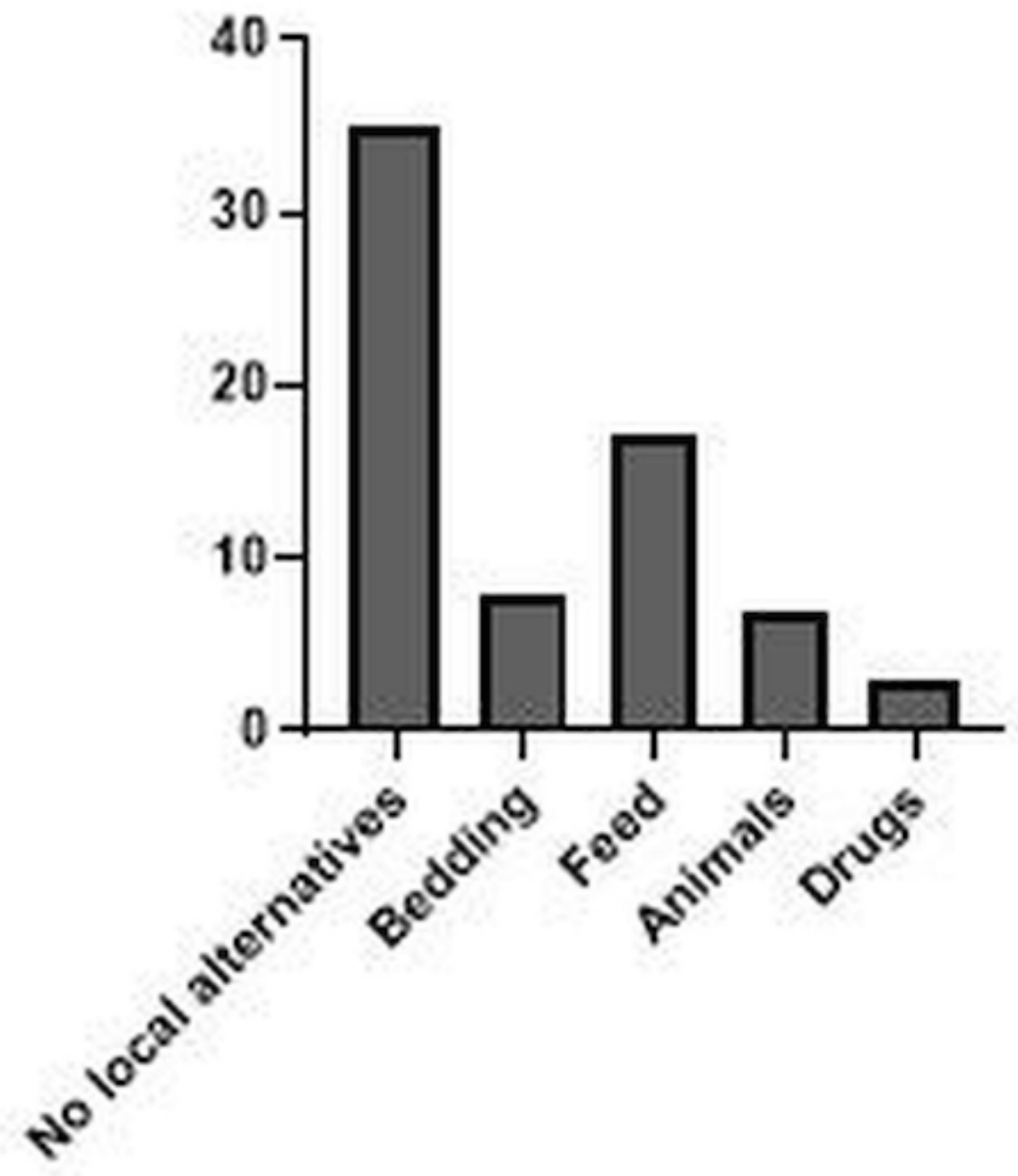

Fig-4: Local Alternative Supplies Availed

Figure 4

Local alternative supplies availed during lock down 


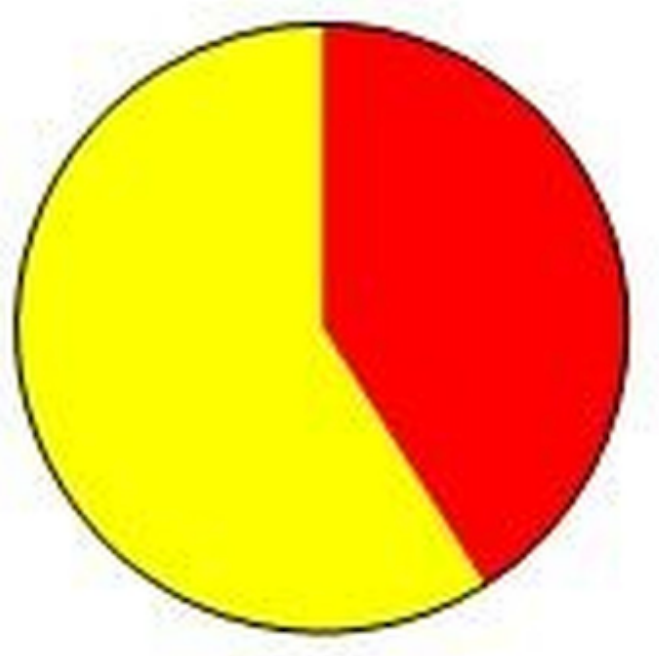

$41.07 \%$ (23) Euthanasia to optimize resources

$\square 58.82 \%(33)$ No euthanasia

Fig-5: Euthanasia of animals to manage resources

Figure 5

Pie diagram representing Euthanesia of animals to manage resources

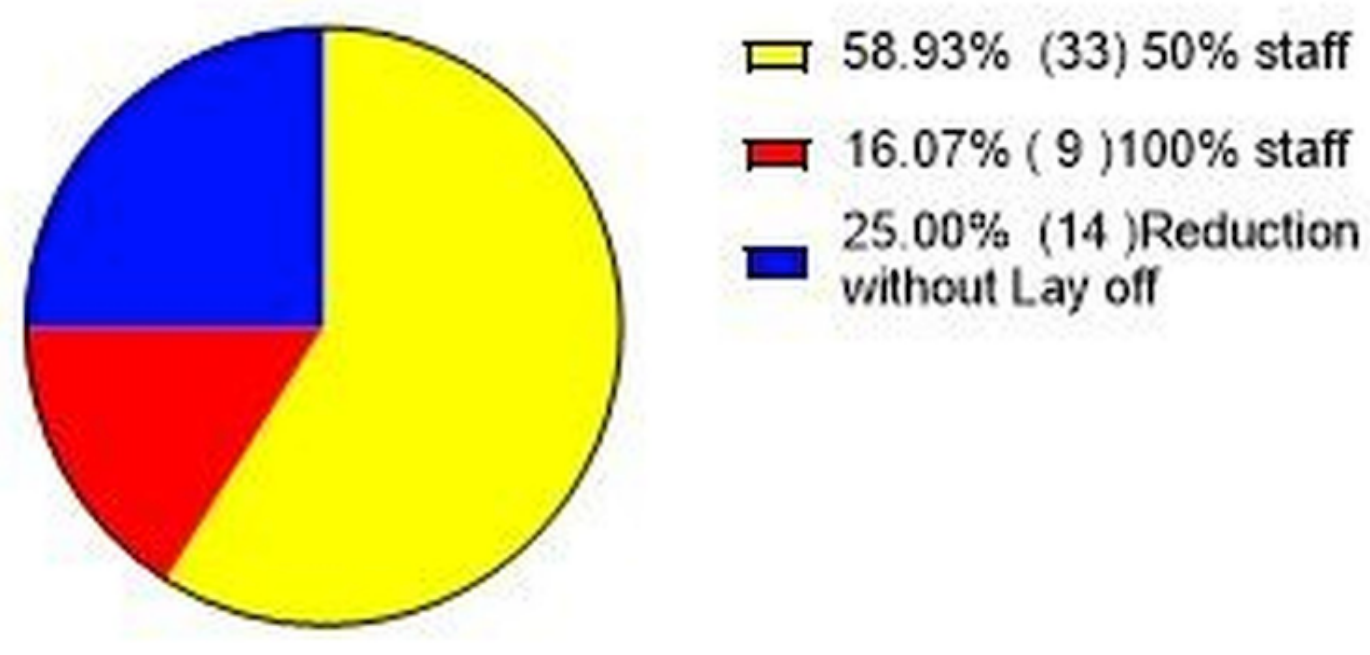

Fig-6: Percentage of manpower available for service

Figure 6 


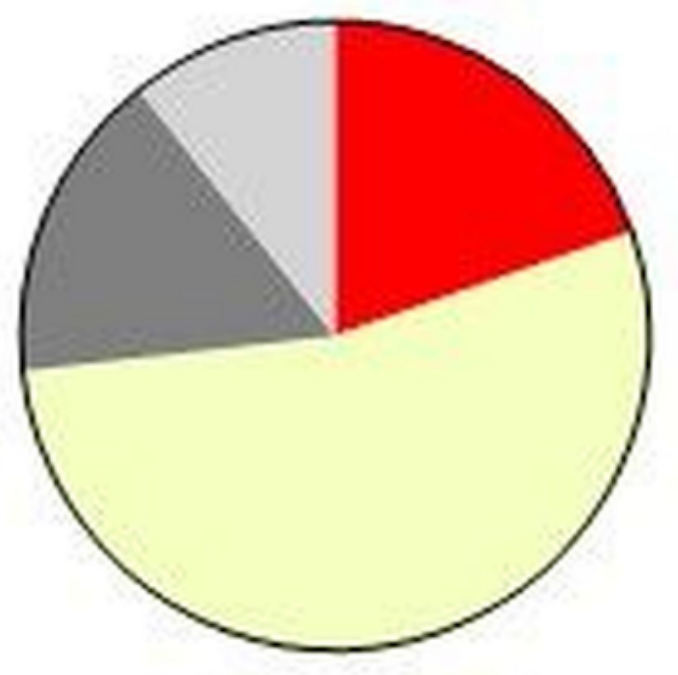

\# $19.64 \% 11$ Completely stopped $\square 53.57 \% 30$ Reduced

$\square 16.07 \% 9$ Did not reduce

曰 $10.71 \% 6 \mathrm{NA}$

\section{Fig-7: Reduction of Breeding of Animals}

\section{Figure 7}

Pie diagram representing percentage of reduction of animals during this period

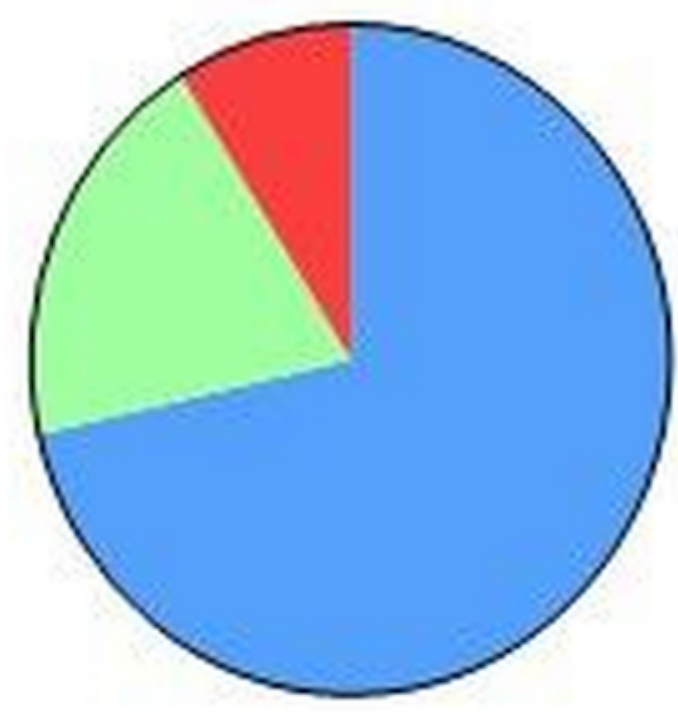

$\square 76.19 \%$ Full-time Veterinary Care

$\square 19.64 \%$ On-Call Veterinary Care

$\square 8.93 \%$ Not Available

Fig-8: Veterinary Care

Figure 8 
Pie diagram representing percentage of veterinary care available during this period.

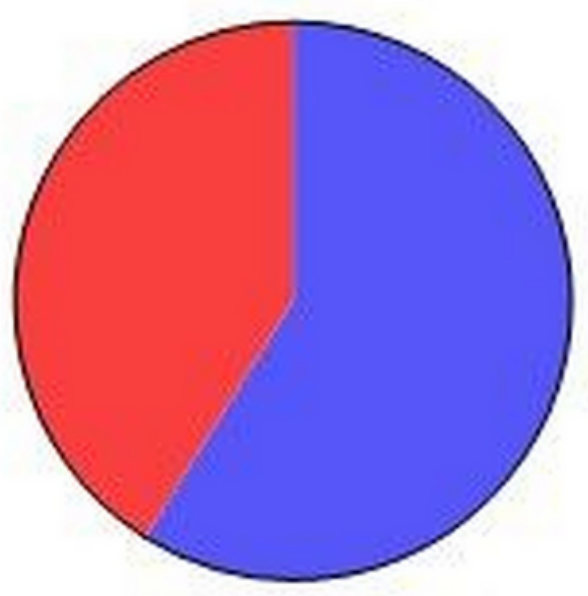

$58.93 \% 33$ Online IAEC Meetings Held $41.07 \% 23$ IAEC meetings Not held

\section{Fig-9: Online IAEC Meetings held during Lockdown}

\section{Figure 9}

Pie diagram representing percentage of online IAEC meeting held during this period

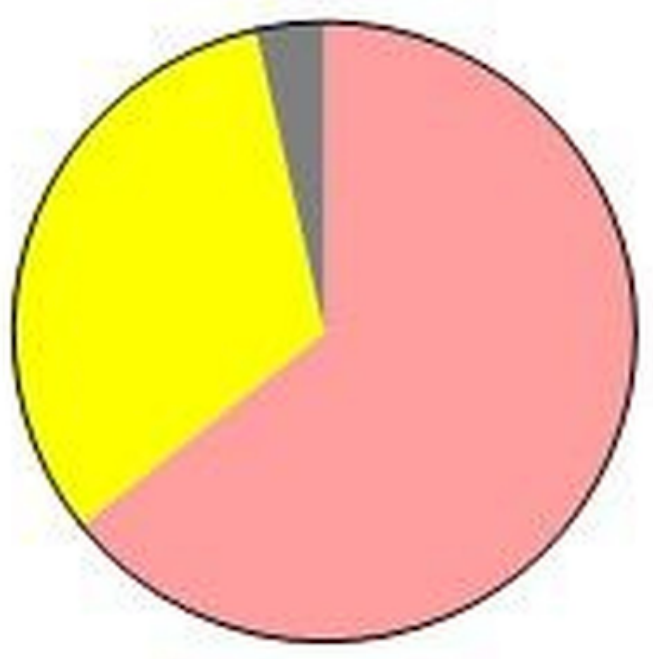

घ $64.29 \% 36$ Morbidity obsenved $\square 32.14 \% 18$ No morbidity observed $\square 3.57 \% 2$ Not answered

Fig-10: Morbidity observed in animal colonies

Figure 10

Pie diagram representing percentage of Morbidity observed during this period 


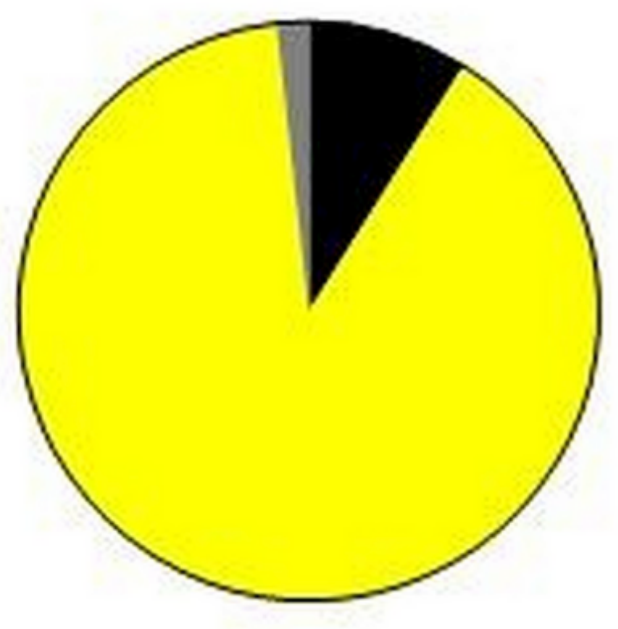

- $8.93 \% 5$ Mortality observed

口 $89.29 \% 50$ Mortality not observed

$\square 1.79 \% 1$ Not Answered

\section{Fig-11: Mortality observed in Animal Colonies}

Figure 11

Pie diagram representing percentage of Mortality observed during this period 